\title{
A NEW EXTRAGRADIENT METHOD FOR PSEUDOMONOTONE VARIATIONAL INEQUALITIES
}

\author{
MUHAMMAD ASLAM NOOR
}

\begin{abstract}
In this paper, we consider and analyze a new extragradient method for solving pseudomonotone variational inequalities. The new method converges for pseudomonotone Lipschitz continuous operators, which is a weaker condition than monotonicity. The new iterative method includes the extragradient method as a special case. Our proof of convergence is very simple as compared with other methods.
\end{abstract}

Mathematics subject classification (2000): 49J40, 90C33.

Key words and phrases: Variational inequalities, extragradient method, fixed-point, convergence.

\section{REFERENCES}

[1] C. Baiocchi And A. CAPElo, Variational and Quasi-Variational Inequalities, J. Wiley and Sons, New York, 1984.

[2] D. P. Bertsekas And J. Tsitsiklis, Parallel and Distributed Computation: Numerical Methods, Prentice-Hall, Englewood Cliffs, New Jersey, 1989.

[3] R. W. Cottle, F. Giannessi And J. L. Lions, Variational Inequalities and Complementarity Problems: Theory and Applications, J. Wiley and Sons, New York, 1980.

[4] F. Giannessi AND A. MaUgeri, Variational Inequalities and Network Equilibrium Problems, Plenum Press, New York, 1995.

[5] M. Aslam NoOR, A modified projection method for monotone variational inequalities, Appl. Math. Letters 12, 83-87, (1999).

[6] M. Aslam Noor, Some recent advances in variational inequalities, Part I, basic concepts, New Zealand J. Math. 26, 53-80, (1997).

[7] M. ASLAM NoOR, Some recent advances in variational inequalities, Part II, other concepts, New Zealand J. Math. 26, 229-255, (1997).

[8] M. Aslam NooR, Some algorithms for general monotone mixed variational inequalities, Mathl. Computer Modelling, 19 (7), 1-9, (1999).

[9] M. ASLAM NOOR, Modified projection method for pseudomonotone variational inequalities, Appl. Math. Letters 15, 315-320, (2002).

[10] M. PATRIKSSON, Nonlinear Programming and Variational Inequalities: A Unified Approach, Kluwer Academic Publishers, Dordrecht, 1998.

[11] G. StamPaCchia, Formes bilineaires coercivities sur les ensembles convexes, C. R. Acad. Sci. Paris 258, 4413-4416, (1964).

[12] Y. WANG, N. XIU AND C. WANG, A unified framework of extragradient-type methods for pseudomonotone variational inequalities, J. Optim. Theory Appl. 111, 641-656, (2002). 